\title{
Laser induced backside wet etching of fused silica: absorption coefficient dependence
}

\author{
Cs. Vass, B. Hopp", T. Smausz \\ Department of Optics and Quantum Electronics, University of Szeged, H-6720 Szeged, Dóm tér 9, Hungary \\ e-mail: vasscs@neptun.physx.u-szeged.hu \\ "Research Group on Laser Physics of the Hungarian Academy of Sciences, H-6720 Szeged, Dóm tér 9, Hungary
}

\begin{abstract}
The micromachining process of transparent materials by laser induced backside wet etching (LIBWE) was investigated. Fused silica targets were irradiated by an ArF excimer laser at 2.14 $\mathrm{J} / \mathrm{cm}^{2}$ fluence and naphthalene solved in methyl-methacrylate with different concentrations were used as absorbing liquid. The absorption coefficient of these solutions was measured by a planoconcave microcuvette and it found to be between 39426 and $623501 / \mathrm{cm}$ depending on the concentration of naphthalene. It was demonstrated that the etch rate depends on the absorption coefficient linearly, while the roughness does not. The dependence of the etch rate can be explained as follows. The absorbed energy in the interface of the solution and the fused silica increases when increasing the absorption coefficient resulting in higher temperature liquid layer at the surface of the fused silica causing higher etch rate.
\end{abstract}

\section{Introduction}

Machining by laser beam of the materials transparent at the laser's wavelength is difficult because of their low absorption. This problem can be eliminated by the LIBWE technique.

The laser-induced backside wet etching (LIBWE) is a new method for processing of UVtransparent dielectric materials. Well-defined, debris and microcracks free micropatterns can be produced by this technique. During this procedure the sample is in contact with a liquid reagent having high absorption coefficient at the applied wavelength. The liquid is irradiated through the sample and the temperature of the first few $\mu \mathrm{m}$ thick layer of fluid becomes higher than the melting point of the solid target due to the laser irradiation. Bubbles forming in this hot liquid 
volume attack the surface of the sample and material removal occurs. The expanded bubbles were observed by X. Ding et al. [2]. J. Wang and co-workers explained the LIBWE with the high temperature of the solution, which is due to the multiphotonic absorption of pyrene (pyrene was soluted in acetone) [3-4]. R. Böhme et al. used pyrene in 3 solvent having different concentrations and investigated how the etch rate depends on these solutions and the applied laser fluence [5]. We investigated how the etch rate and the morphology of the processed surface depend on the absorption coefficient of the liquid reagents. The concentration of our solutions was changed to produce different absorption coefficients.

\section{Experimental}

\subsection{Absorption coefficient measurement}

The absorbing liquids were solutions of naphthalene in methyl-methacrylate at different concentrations: $0,0.21,0.43,0.85$ and $1.71 \mathrm{~mol} / \mathrm{dm}^{3}$, respectively. The absorption coefficient of these reagents at the wavelength of $\mathrm{ArF}$ excimer laser $(193 \mathrm{~nm})$ used for the irradiation of the sample is important in our experiments. Measuring the absorption coefficient on the conventional way (using spectrophotometer) at this wavelength is not possibility, because the liquids have high absorption coefficient and we can not produce so liquid layers thin enough to transmit sufficient light for detection. Therefore this parameter was measured with a planoconcave microcuvette (Fig.1.).

The plano-concave microcuvette was illuminated by a homogeneous part of the low energy ArF excimer laser beam. The transmitted beam intensity can be written in Gaussian profile. Measuring the transmitted intensity, the absorption coefficient $(\alpha)$ can be determinated from the FWHM of the Gaussian profile: $\alpha=\frac{2 R}{\text { FWHM }^{2}} 4 \ln 2$, where $R$ is the radius of the curvature of the applied lens $(\mathrm{R}=19.67 \mathrm{~mm})$. A Gaussian profile of the transmitted light in the case of 0.85 
$\mathrm{mol} / \mathrm{dm}^{3}$ solution can be seen in Fig. 2. It was found that the $\alpha$ increases till $0.43 \mathrm{M}$ $\left(\mathrm{M}=\mathrm{mol} / \mathrm{dm}^{3}\right)$ concentration, and decreases at higher concentration (Fig. 3.). We suppose that this effect can be due to the chemical interactions between the naphthalene and the methylmethacrylate molecules.

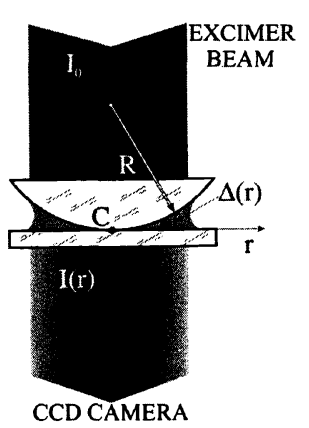

Fig. 1. Scheme of the planoconcave microcuvette: $C$, the central point of the cuvette; $I_{0}$, the intensity of the incoming light; $I(r)$, the value of the transmitted Gaussian intensity profile; $\Delta(r)$, the liquid layer thickness

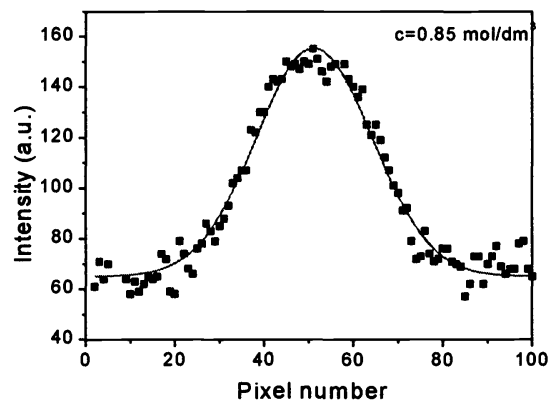

Fig. 2. The cross section of the transmitted light $\left(\mathrm{c}=0.85 \mathrm{~mol} / \mathrm{dm}^{3}\right)$ and the fitted Gaussian function

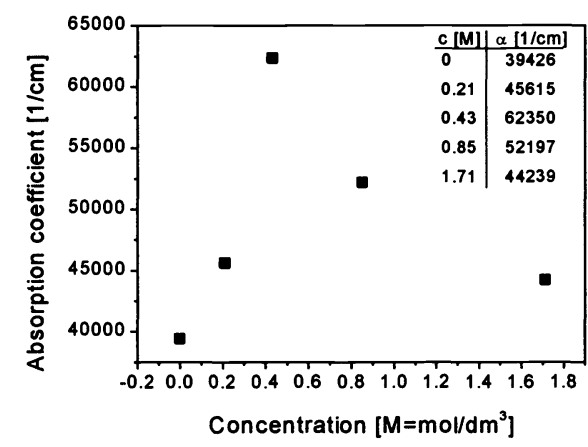

Fig. 3. Absorption coefficient versus concentration. $\alpha$ increases till $0.43 \quad M$ $\left(M=\mathrm{mol} / \mathrm{dm}^{3}\right)$ concentration, and decreases at higher concentration. We suppose that this effect can be due to the chemical interactions between the naphthalene and the methylmethacrylate molecules.

\subsection{Dependence of the etch rate on the absorption coefficient}

The experimental set-up used for the etching is shown in Fig 4. An ArF excimer laser (FWHM=20 ns) was used for irradiation the samples. The investigated transparent material was fused silica. The liquid reagents were the solutions mentioned above. The applied fluence was constant $\left(\mathrm{F}=2.14 \mathrm{~J} / \mathrm{cm}^{2}\right)$ during our measurements. A blade was placed onto the surface of the fused silica to shut the half of the laser beam to make possible accurate measurement of the etch depth. The etch depths were measured by TopoMetrix 2000 atomic force microscope. The numbers of the etching laser pulses were 250,500 and 750 , respectively. The etch rates were calculated and it was found, that these are independent of the number of pulses. The etch rate of fused silica versus absorption coefficient is shown in Fig. 5. It can be seen clearly that this depends on the absorption coefficient of the liquid reagents linearly. 


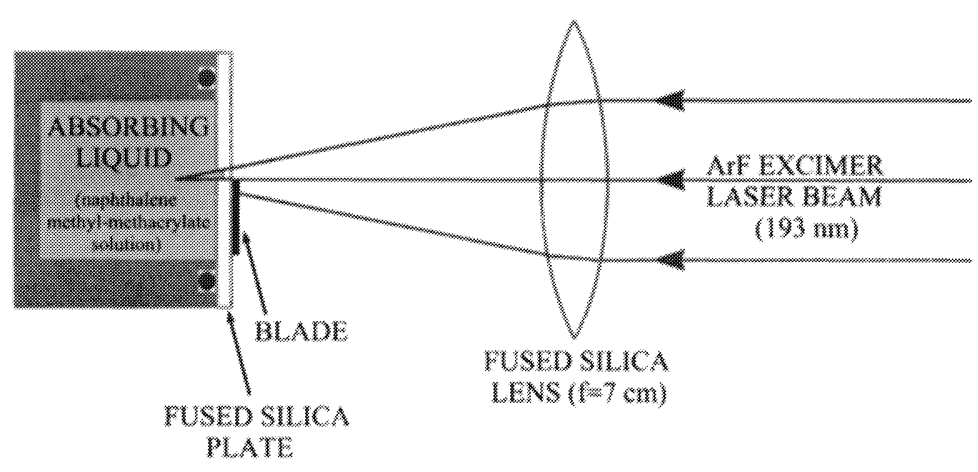

Fig. 4. Schematic diagram of the experimental setup for the etching

\subsection{Morphological study}

Fig. 6. shows the AFM pictures of an edge and the bottom of an etched hole.

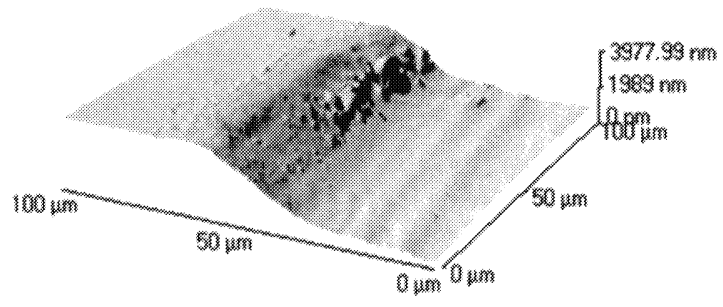

Fig. 6. a. An edge generated $c=0.43 \mathrm{M}$ and 750 pulses

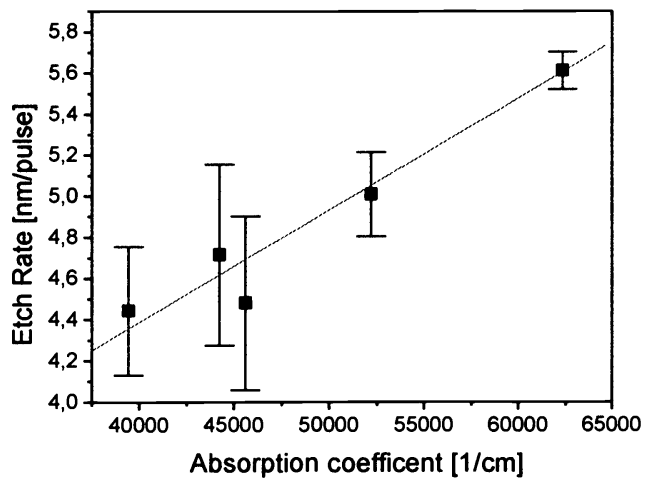

Fig. 5. The etch rate versus absorption coefficient

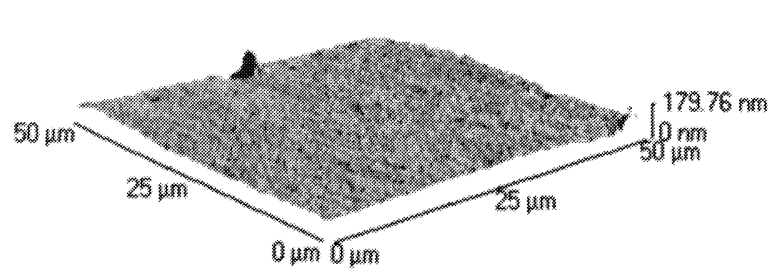

Fig. 6. b. the bottom of the etched hole generated $c=0.85 \mathrm{M}$ and 250 pulses

The edge is not sharp because the blade was placed onto outer surface of the fused silica (Fig.

4.). The roughness of the treated surfaces is characterised by the $R_{a}$ parameter $\left(R_{a}=\frac{1}{L} \int_{o}^{L} f f(x) \mid d x\right)$. We found that $R_{a}$ is independent of the $\alpha$. The average value of $R_{a}$ was $27 \mathrm{~nm}$, which is much smaller than the etch depth. The $R_{a}$ parameter versus absorption coefficient is shown in the Fig. 7.

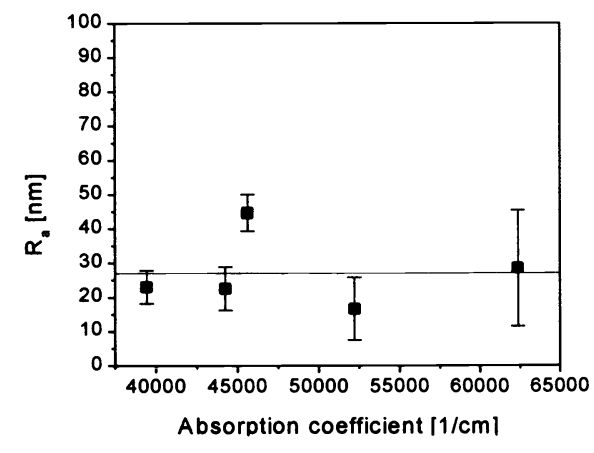

Fig. 7. $R_{a}$ versus absorption coefficient.

\section{Conclusions}

The mechanism of LIBWE can be explained as follows. Due to the high absorption coefficient of the naphthalene - methyl-methacrylate solution, the UV photons are absorbed in a few $\mu \mathrm{m}$ thin liquid layer contacting the fused silica sample. Therefore the temperature increases dramatically above the melting point of the fused silica in this layer and the surface of the sample melts, the 
liquid vaporizes, bubbles form in the boiling liquid volume and expand suddenly. The high pressure generated by these expanding bubbles causes strong mechanical effects, which damage the surface of the fused silica.

Acknowledgements The authors gratefully acknowledge the financial support of foundation OTKA (T34825, TS 040759), the Hungarian Ministry for Culture and Education (NKFP 3/064/2001) and PRCH Student Science Foundation.

\section{References}

[1] D. Bäuerle, Laser Processing and Chemistry, Springer, Berlin, 2000.

[2]X.Ding,Y,Kawaguchi,H.Niino,A.Yabe,Appl.Phys.A,DOI 10.1007/s00339-002-1453-1 (2002)

[3] J. Wang, H. Niino, A. Yabe, Appl. Surf. Sci. 154-155 (2000) 571-576

[4] J. Wang, H. Niino, A. Yabe, Appl. Phys. A, 69 (Suppl.) S271-S273 (1999)

[5] R. Böhme, A. Braun, K. Zimmer, Appl. Surf. Sci. 186 (2002) 276-281 